J. Clin. Chem. Clin. Biochem.

Vol. 16, 1978, pp. 621-624

\title{
Verhalten von Gesamtöstriol, nichtkonjugiertem Östriol, Human Placenta Lactogen und Oxyto- cinase im Serum sowie der Gesamtöstrogene im Urin von schwangeren Frauen nach Gabe von Beta- methason
}

\author{
Von E. Knoll, A. Kayser und H. Wisser ${ }^{1}$ )
}

\begin{abstract}
Abteilung für Klinische Chemie und Abteilung für Geburtshilfe und Gynäkologie am Robert-Bosch-Krankenhaus, Stuttgart
\end{abstract}

(Eingegangen am 13. März/27. Juli 1978)

Zusammenfassung: Die prophylaktische Therapie von Schwangeren im letzten Trimenon mit Betamethason zur Vermeidung des Atemnotsyndroms der Neugeborenen führt zu Veränderungen einiger biochemischer Parameter, die zur Kontrolle der fetoplacentaren Einheit verwendet werden. Bei 12 Schwangeren wurde der Einfluß von Betamethason auf die Konzentrationen von Gesamtöstriol, nichtkonjugiertem Östriol, Human Placenta Lactogen und Oxytocinase im Serum sowie auf die Ausscheidung der Gesamtöstrogene im Urin untersucht. Beide Serum-ÖstriolFraktionen und die Gesamtöstrogene im Urin fallen innerhalb von 1 bis 2 Tagen auf 50-60\% der Ausgangswerte ab. Dies ist als Folge der Suppression der fetalen Hypophysen-Nebennierenrindenachse durch Betamethason aufzufassen, wodurch die Biogenese des Östriol-Präkursors 16- $\alpha$-Hydroxydehydroepiandrosteronsulfat vermindert wird. Die Zeitdauer bis zum Wiedererreichen bzw. Überschreiten der Basiswerte unterliegt starken interindividuellen Schwankungen: 11 Tage für das nichtkonjugierte Östriol, 13 Tage für die Gesamtöstrogene im Urin und 18 Tage für das Gesamtöstriol im Serum. Die rein placentaren Parameter Human Placenta Lactogen und Oxytocinase zeigen keine Beeinflussung durch die Betamethason-Therapie und behalten ihren für diese Schwangerschaftsperiode typischen leicht ansteigenden Verlauf bei.

Concentrations of total oestriol, non-conjugated oestriol, human placental lactogen and oxytocinase in the serum, and total oestrogens in the urine of pregnant women, following the administration of betamethasone

Summary: Prophylactic therapy with betamethasone is used during the last three months of pregnancy, for the prevention of the respiratory distress syndrome in the new born child. This results in changes in some biochemical parameters, which are normally used to monitor the foetoplacental unit. The influence of betamethasone on the concentrations of total oestriol, non-conjugated oestriol, human placental lactogen and oxytocinase in the serum, and on the urinary excretion of total oestrogens was investigated. Both of the serum oestriol fractions and the total urinary oestrogens decrease to $50-60 \%$ of their starting values within 1-2 days after the onset of betamethasone therapy. This is thought to be due to the suppression of the foetal hypophysis-adrenal cortex interaction by betamethasone, which results in a decrease in the biogenesis of the oestriol precursor, 16- $\alpha$-hydroxy-dehydroepiandrosterone sulphate. There are marked individual variations in the time taken to re-establish or to exceed the basal values: 11 days for the non-conjugated oestriol, 13 days for the total urinary oestrogens, and 18 days for the total oestriol in the serum. The purely placental parameters, human placental lactogen and oxytocinase were not influenced by betamethasone therapy; they showed their usual slight increase, which is typical for this period of pregnancy.

\section{Einführung}

1972 wirden von Liggins \& Howie (1) zur Prophylaxe des Atemnotsyndroms von Neugeborenen die pränatale Therapie mit hochdosierten Glucocorticoiden eingeführt. Die Glucocorticoide induzieren eine vermehrte Synthese des pulmonalen fetalen ,Surfactants“ (2). Schwenzel et al. (3) publiżierten 1975 ihre Ërfahrungen mit der pränata- len Beeinflussung der kindlichen Lungenreife durch Betamethason. Bei einer Gesamtzahl von 185 Schwangeren, von denen 76 mit Betamethason behandelt wurden, traten bei den Neugeborenen in der Betamethason-Gruppe wesentlich seltener schwere Atemstörungen auf als im

1) Diese Arbeit wurde unterstützt aus Mitteln der Robert-BoschStiftung, Stuttgart. 
nicht behandelten Kontrollkollektiv. In der behandelten Gruppe starb nur ein Neugeborenes am Atemnotsyndrom, in der Kontrollgruppe starben dagegen 11 Kinder. Inzwischen wird die Glucocorticoid-Therapie in vielen Kliniken mit Erfolg durchgeführt.

Seit längerer Zeit ist bekannt, daß die Östrogen-Ausscheidung schwangerer Frauen nach Gabe von Cortison und Prednison abfällt $(4,5,6,7)$, die Pregnandiol-Ausscheidung dagegen unbeeinflußt bleibt $(6,7)$. Dieses Absinken der Östrogene ist eine Folge der Suppression der fetalen Nebennierenrinde durch die Glucocorticoide. Hierbei wird insbesondere der Hauptpräkursor des Östriols, das 16- $\alpha$-Hydroxydehydroepiandrosteronsulfat, supprimiert $(8,9)$. Aus den absinkenden Östrogenkonzentrationen kann im allgemeinen keine Gefährdung des Feten abgeleitet werden, doch fällt hiermit der wohl wichtigste biochemische Parameter als Beurteilungskriterium des $\mathrm{Zu}$ standes von Fet und Placenta für einige Tage aus. Ziel dieser Untersuchung sollte sein, Ausmaß und Dauer der Beeinflussung von nichtkonjugiertem und Gesamtöstriol, Human Placenta Lactogen und Oxy tocinase im Serum und der Gesamtöstrogene im Urin durch Betamethason festzustellen.

\section{Versuchsbeschreibung}

12 schwangere Frauen wurden zur Vermeidung eines drohenden Atemnotsyndroms der Neugeborenen zwischen der 28. und 36. Schwangerschaftswoche mit Betamethason behandelt. Die Probandinnen erhielten in der Regel 2 mal je 1 Ampulle CelestanDepot $^{2}$ ) im Abstand von 12 Stunden. Zur Ermittlung der Ausgangswerte wurden an jeweils 3 Tagen vor der Celestan-Gabe 24-Stunden-Urine gesammelt und zwischen 8.00 und $10.00 \mathrm{Uhr}$ Blutproben entnommen. Die täglichen Urinsammlungen und Blutentnahmen wurden bis 20 Tage nach der Celestan-Gabe fortgesetzt. Bezüglich der Ernährung wurde den Frauen keine Auflage gemacht. Während der Versuchsdauer wurden keine zusätzlichen Medikamente gegeben. Die Serum- und Urinproben wurden - falls sie nicht sofort analysiert wurden - bis zur Analyse bei $-30^{\circ} \mathrm{C}$ aufbewahrt.

\section{Methoden}

Nichtkonjugiertes und Gesamtöstriol wurden radioimmunologisch bestimmt. Das dabei verwendete Antiserum war hochspezifisch und wurde durch Immunisierung von Neuseeland-Kaninchen mit Östriol-6-carboxymethyloxim, das an Rinder-SerumAlbumin gekoppelt war, gewonnen (10). Nichtkonjugiertes Östriol wurde aus dem Serum mit Diäthyläther extrahiert.

Zur Bestimmung von Gesamtöstriol im Serum wurden die Östriol-Konjugate durch 1-stündiges Inkubieren mit einem Arylsulfatase $/ \beta$-Glucuronidase-Gemisch ${ }^{3}$ ) bei $50^{\circ} \mathrm{C}$ hydrolysiert. Danach verläuft die Analyse analog der Bestimmung von nichtkonjugiertem Östriol. Human Placenta Lactogen wurde ebenfalls radioimmunologisch bestimmt ${ }^{4}$ ).

Die Analyse der Gesamtöstrogene im Urin erfolgte photometrisch nach dem Kober-Ittrich-Bradlow-Verfahren (11). Die Stéroidkonjugate wurden aus dem Urin mit Amberlite XAD-2 ${ }^{5}$ ) abgetrennt.

\footnotetext{
2) $4 \mathrm{mg}$ Betamethasondinatriumphosphat $+3 \mathrm{mg}$ Betamethasonacetat.

4) Firma Boehringer, Mannheim.

4) Testkit Firma Amersham Buchler.

5) Serva, Heidelberg.
}

Die Bestimmung der Oxy tocinase wurde kinetisch mit einem Fast-Analyzer durchgeführt. Die Einzelheiten der Methodik sind an anderer Stelle publiziert (12).

\section{Ergebnisse und Diskussion}

Tabelle 1 zeigt die Präzision der angewandten Methoden. Die Variationskoeffizienten der Streuung von Tag zu Tag liegen bei den verschiedenen Verfahren zwischen 5 und $11 \%$.

Die Ergebnisse unserer Untersuchung sind in Abbildung 1 graphisch dargestellt.

Als Basalwerte wurden die Mittelwerte der 5 Parameter an den letzten 3 Tagen vor Gabe des Medikamentes verwendet. An den Tagen nach der Betamethason-Gabe sind die bei allen 12 Versuchspersonen gemessenen Werte als mittlere prozentuale Abweichungen von den Basalwerten ( \pm 1 Standardabweichung) zusammengefaßt.

Übereinstimmend fallen Gesamtöstrogene im Urin und Serum-Östriol auf 50-60\% der Basiswerte ab. Nichtkonjugiertes Östriol zeigt den steilsten Abfall und erreicht schon am Tag nach der Betamethason-Gabe ein Minimum von $-43 \%$ der Ausgangskonzentration. Dies steht in Übereinstimmung mit den Befunden von Negulescu et al. (13), die die Veränderungen von Plasma-Östriol nach Betamethason-Injektion (12 mg, i. m.) in kurzen Zeitabständen untersuchten und bereits 3 Stunden nach der Betamethason-Injektion einen maximalen Abfall von 69\% bei normalen und $33 \%$ bei pathologischen Schwangerschaften fanden. Wir fanden ein um so stärkeres Absinken der Absolutwerte, je höher die Ausgangswerte waren, doch waren die prozentualen Werte unabhängig von den Basiskonzentrationen.

11 Tage nach der Betamethason-Gabe erreichte bzw. überschritt das nichtkonjugierte Östriol die Ausgangswerte vor Therapiebeginn. Wie aus den Standardabweichungen zu ersehen ist, unterlag dieser Zeitpunkt starken interindividuellen Schwankungen, die beim Gesamtöstriol im Serum und den Gesamtöstrogenen im Ürin noch wesentlich stärker waren.

Diese beiden Parameter erreichten erst am 2. Tag nach der Celestan-Gabe ein Minimum und blieben dann lange supprimiert. Das Gesamtöstriol im Serum erreichte erst am 18. Tag die Basiswerte, und die Gesamtöstrogene im Urin überschritten die Ausgangswerte ebenfalls erst am 13. Tag. Dies deckt sich mit den Befunden von Ohrlander et al. (14), die über ein Wiedererreichen der Basiswerte von Östriol im Urin erst in der 3. Woche nach Betamethason-Gabe $(3 \times 12 \mathrm{mg})$ berichten. Gestützt werden diese Ergebnisse auch durch die Tatsache, daß die Suppression der Hypophysen-Nebennierenrindenachse bis zu 3 Wochen anhält, wie aus Messungen des mütterlichen Plasma-Cortisols hervorgeht (14). Der sogenannte „Östrogensturz“ ist anscheinend sowohl für die beiden gemessenen ÖstriolParameter im Serum als auch für die Gesamtöstrogene im Urin proportional zur Basiskonzentration, d.h. der 
Tab. 1. Ergebnis der Präzisionskontrolle der 5 verwendeten Bestimmungsverfahren.

\begin{tabular}{|c|c|c|c|c|c|}
\hline Substanz & $\begin{array}{l}\text { Gesamtöstriol } \\
\text { im Serum }\end{array}$ & $\begin{array}{l}\text { nichtkonjugiertes Östriol } \\
\text { im Serum }\end{array}$ & $\begin{array}{l}\text { Human Placenta } \\
\text { Lactogen im Serum }\end{array}$ & $\begin{array}{l}\text { Oxytocinase } \\
\text { im Serum }\end{array}$ & $\begin{array}{l}\text { Gesamtöstrogene } \\
\text { im Urin }\end{array}$ \\
\hline Präzision & $\begin{aligned} \bar{x} & =148,3 \mu \mathrm{g} / 1 \\
\mathrm{~s} & =7,65 \mu \mathrm{g} / 1 \\
\mathrm{VK} & =5,2 \% \\
\mathrm{n} & =11\end{aligned}$ & $\begin{aligned} \bar{x} & =12 \mu \mathrm{g} / 1 \\
\mathrm{~s} & =0,87 \mu \mathrm{g} / 1 \\
\mathrm{VK} & =7,2 \% \\
\mathrm{n} & =14\end{aligned}$ & $\begin{aligned} \bar{x} & =7,8 \mathrm{mg} / 1 \\
\mathrm{~s} & =0,39 \mathrm{mg} / 1 \\
\mathrm{VK} & =5 \% \\
\mathrm{n} & =20\end{aligned}$ & $\begin{aligned} \bar{x} & =70 \mathrm{U} / 1 \\
\mathrm{~s} & =4,2 \mathrm{U} / 1 \\
\mathrm{VK} & =6 \% \\
\mathrm{n} & =20\end{aligned}$ & $\begin{aligned} \bar{x} & =20,5 \mathrm{mg} / 1 \\
\mathrm{~s} & =2,36 \mathrm{mg} / \mathrm{l} \\
\mathrm{VK} & =11,5 \% \\
\mathrm{n} & =14\end{aligned}$ \\
\hline
\end{tabular}

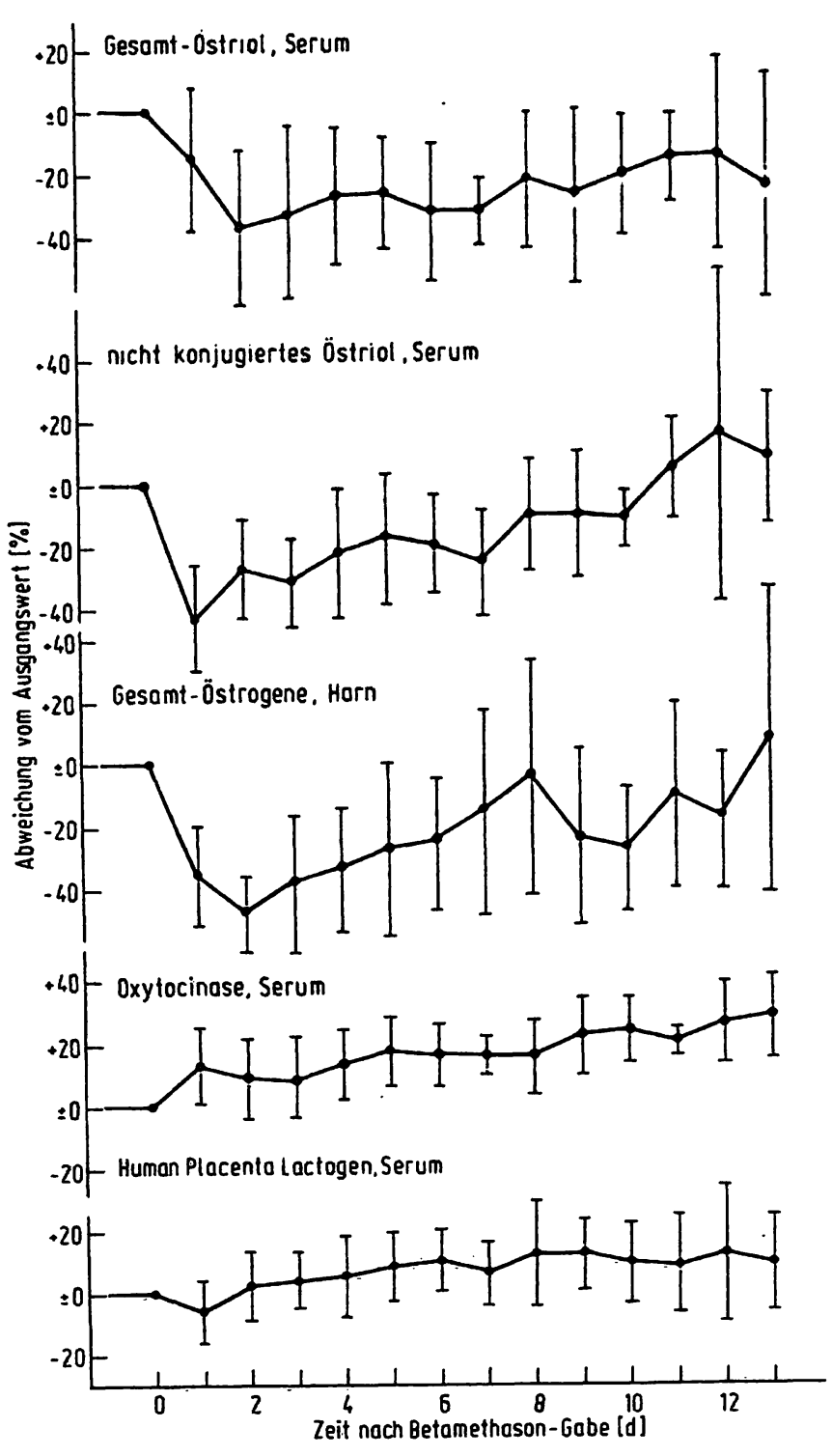

Abb. 1. Gesam töstriol, nichtkonjugiertes Östriol, Human Placenta Lactogen und Oxy tocinase im Serum sowie Gesamtöstrogene im Urin nach Gabe von Betamethason als prozentuale Abweichungen von den Ausgangswerten. Die.einzelnen Werte sind jeweils Mittelwerte von 12 Versuchspersonen, die senkrechten Linien stellen die Standardabweichung innerhalb dieser Patientinnengruppe dar.

Absolutwert des „Östrogensturżes“ ist umso größer, je höher die jeweilige Basiskonzentration vor Therapiebeginn lag.

Im Gegensatz zu den Östrogenen wurden die Konżentrationsverläufe von Human Placenta Lactogen und Oxy-
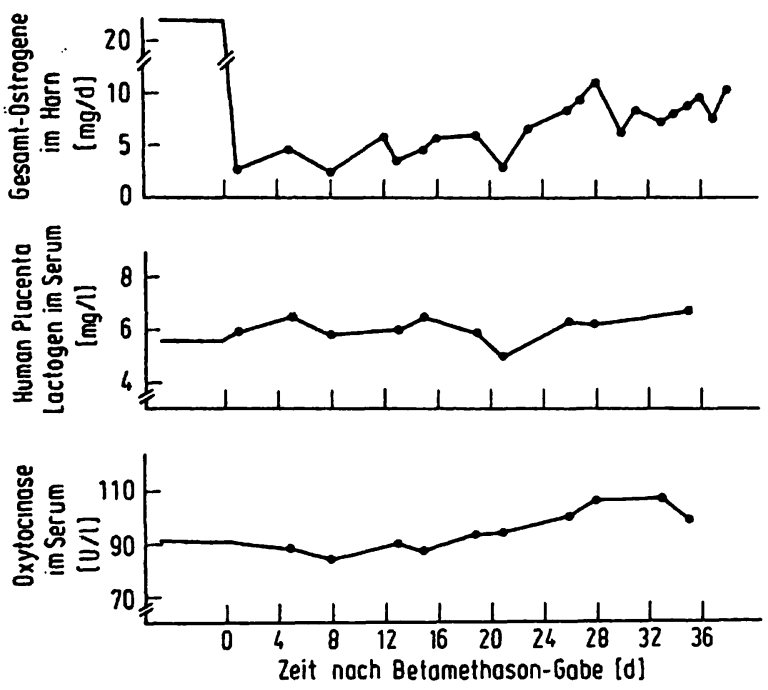

Abb. 2. Verhalten von Human Placenta Lactogen und Oxytocinase im Serum sowie der Gesamtöstrogene im Urin nach Gabe von Celestan-Depot $(3 \times 1$ Ampulle) bei einer $\mathrm{Pa}$ tientin in der 31 . Schwangerschaftswoche.

tocinase im Serum durch die Betamethason-Therapie nicht beeinflußt. $D a$ beide als rein placentare Parameter nicht wie Östriol aus adrenalen Präkursoren entstehen, kann sich die Suppression der fetalen Hypophysen-Nebennierenrindenachse nicht auf ihre Synthese auswirken. Auch auf die Pregnandiol-Ausscheidung im Urin hat eine Glucocorticoid-Therapie in der Schwangerschaft keinen Einfluß (7), da Progesteron, der Präkursor dieser Substanz, überwiegend in der Placenta aus Pregnenolon entsteht.

Ein Beispiel des unterschiedlichen Verhaltens der rein placentaren Parameter Human Placenta Lactogen und Oxytocinase einerseits sowie der Östrogene andererseits, zeigt Abbildung 2.

Eine Frau in der 31. Schwangerschaftswoche erhielt $3 \times 1$ Ampulle Celestan-Depot im Abstand von jeweils 12 Stunden. Während die Gesamtöstrogene im Urin von $22 \mathrm{mg} / 24 \mathrm{~h}$ auf $2-3 \mathrm{mg} / 24 \mathrm{~h}$ abfielen und bis zur Entbindung in der 36 . Schwangerschaftswoche die Basiswerte nicht wieder erreichten, behielten die Human Placenta Lactogen- und Oxytocinase-Werte eine leicht ansteigende Tenden $z$ bei. 
Unsere Untersuchungen bestätigen, daß die Betamethason-Therapie zur Prophylaxe des Atemnotsyndroms von Neugeborenen infolge Suppression der HypophyșenNebennnierenrindenachse des Feten zu einem 2-3 wöchigen Absinken der Östrogene im Serum bzw. Urin der Mutter führt. Während dieser Zeitspanne können die
Östrogene deshalb nicht zur Beurteilung der Funktion der fetoplacentaren Einheit herangezogen werden. Die Aussagekraft von Human Placenta Lactogen- und Oxytocinase-Bestimmungen zur Beurteilung der Placentafunktion bleibt in dieser Phase erhalten.

\section{Literatur}

1. G. C. Liggins \& R. N. Howie (1972), Pediatrics 50, 515-520.

2. E. Kuss (1976), diese Z. 14, 505-513.

3. W. Schwenzel, H. Jung, H. Lahmann, A. Etzrodt, C. Sticherling, K. Korz, B. Liedtke \& H. Chantraine (1975), Z. Geburtshilfe Perinatol. 179, 45-52.

4. J. C. Warren \& S. G. Cheatum (1967), J. Clin. Endocrinol. $27,433-436$.

5. J. B. Brown, N. A. Beischer \& M. A. Smith (1968), J. Obstet. Gynaecol. Br. Commonw. 75, 819-828.

6. A. M. Driscoll (1969), Br. Med. J. 1, 556-557.

7. R. E. Oakey (1970), J. Obstet. Gynaecol. Br. Commonw. 77, 922-927.

8. H. H. Simmer, D. Tulchinsky, E. M. Gold, M. Frankland, M. Greipel \& A. S. Gold (1974), Am. J. Obstet. Gynecol. 119, 283-296.

9. H. H. Simmer, M. Frankland \& M. Greipel (1975), Am. J. Obstet. Gynecol. 121, 646-652.

10. R. Goebel \& E. Kuss (1974), Geburtshilfe und Frauenheilkunde. 34, 329-338.

11. E. Kuss \& R. Goebel (1976), diese Z. 14, 549-556.

12. H. Wisser, K. Dettmer \& E. Knoll (1976), diese Z. 14, 333337.

13. R. J. Negulescu, J. R. Strecker, C. Lauritzen \& S. Pal (1977), J. Perinat. Med. 5, 120-132.

14. S. A. V. Ohrlander, G. M. Gennser \& L. Grennert (1975), Am. J. Obstet. Gynecol. 123, 228-236.

Dr. rer. nat. E. Knoll

Abteilung für Klinische Chemie

Robert-Bosch-Krankenhaus

Auerbachstr. 110

D-7000 Stuttgart 50 